This is an electronic reprint of the original article. This reprint may differ from the original in pagination and typographic detail.

\author{
Author(s): Isoniemi, Tommi; Johansson, Andreas; Hakala, Tommi; Rinkiö, Marcus; Törmä, Päivi; \\ Toppari, Jussi; Kunttu, Henrik
}

Title: $\quad$ Surface plasmon effects on carbon nanotube field effect transistors

Year: $\quad 2011$

Version:

Please cite the original version:

Isoniemi, T., Johansson, A., Hakala, T., Rinkiö, M., Törmä, P., Toppari, J., \& Kunttu, H. (2011). Surface plasmon effects on carbon nanotube field effect transistors. Applied Physics Letters, 99, 31105. https://doi.org/10.1063/1.3614543

All material supplied via JYX is protected by copyright and other intellectual property rights, and duplication or sale of all or part of any of the repository collections is not permitted, except that material may be duplicated by you for your research use or educational purposes in electronic or print form. You must obtain permission for any other use. Electronic or print copies may not be offered, whether for sale or otherwise to anyone who is not an authorised user. 


\title{
Surface plasmon effects on carbon nanotube field effect transistors
}

\author{
T. Isoniemi, ${ }^{1}$ A. Johansson, ${ }^{1}$ T. K. Hakala, ${ }^{1, a)}$ M. Rinkiö, ${ }^{1,2}$ P. Törmä, ${ }^{2}$ J. J. Toppari, ${ }^{1, b)}$ \\ and $\mathrm{H}$. Kunttu ${ }^{3}$ \\ ${ }^{1}$ Nanoscience Center, Department of Physics, University of Jyväskylä, P.O. Box 35 (YN), FIN-40014 \\ Jyväskylä, Finland \\ ${ }^{2}$ Department of Applied Physics, Aalto University School of Science, P.O. Box 15100, \\ FIN-00076 AALTO, Finland \\ ${ }^{3}$ Nanoscience Center, Department of Chemistry, University of Jyväskylä, P.O. Box 35 (YN), \\ FIN-40014 Jyväskylä, Finland
}

(Received 19 April 2011; accepted 28 June 2011; published online 19 July 2011)

\begin{abstract}
Herein, we experimentally demonstrate surface plasmon polariton (SPP) induced changes in the conductivity of a carbon nanotube field effect transistor (CNT FET). SPP excitation is done via Kretschmann configuration while the measured CNT FET is situated on the opposite side of the metal layer away from the laser, but within reach of the launched SPPs. We observe a shift of $\sim 0.4 \mathrm{~V}$ in effective gate voltage. SPP-intermediated desorption of physisorbed oxygen from the device is discussed as a likely explanation of the observed effect. This effect is visible even at low SPP intensities and within a near-infrared range. (C) 2011 American Institute of Physics. [doi:10.1063/1.3614543]
\end{abstract}

Field effect transistors (FETs) utilizing carbon nanotubes (CNTs) as their conductance channel have possible applications, among others, as chemical ${ }^{1,2}$ and optical sensors ${ }^{3}$ as well as in photoelectronics. ${ }^{4}$ Chemical sensing is generally based on adsorption or desorption of different molecular species that alter the electrical response of semiconducting single-walled (SW) CNTs (Refs. 1 and 5) (as well as graphene $^{6}$ ). In ambient conditions, specifically oxygen and water vapor have been shown to significantly influence CNT FETs, ${ }^{7,8}$ even though most of the oxygen is only physisorbed on the SWCNT via van der Waals interactions. ${ }^{9}$ On the other hand, photoexcitation of a SWCNT, which is especially important for photoelectrical applications, can induce several different effects in its electrical transport properties. ${ }^{3-5}$ While the direct electron-hole generation is the most desired effect in photoelectronics, another usually even more important and widely studied effect is due to photodesorption of atmospheric molecules. ${ }^{5,8,10,11}$ The photodesorption is strongest in the UV range and usually negligible in near-IR. ${ }^{5}$

Surface plasmon polaritons (SPPs) are coupled modes of electromagnetic waves and oscillations of free electrons in a metal surface. They can be considered as two-dimensional light bound to a metal-dielectric interface, however, with all the properties modified by the subwavelength confinement of these optical fields. ${ }^{12,13}$ Thus, SPPs offer fascinating prospects for photoelectronics. For example, a huge field enhancement near the interface produced by the confinement has been widely utilized in surface enhanced Raman spectroscopy. ${ }^{14}$ In this letter, we utilize SPPs to "illuminate" the CNT FET, which leads to a significant effect on its charge transfer properties. Surface plasmons have been shown to cause desorption on metal surfaces. ${ }^{15}$ Here, the observed effect can also be explained by the desorption of oxygen induced by the SPP illumination. Contrary to far-field induced photodesorption,

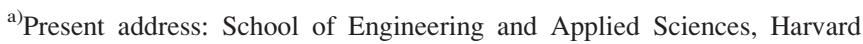
University, Cambridge, Massachusetts 02138, USA.

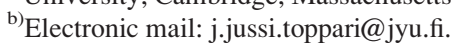

the effect observed here is visible at low intensities and even in near-IR.

Schematics of the experimental setup and an AFM image of the CNT FET measured are shown in Fig. 1. SPPs are launched in the Kretschmann configuration, where the incoming light at the resonant angle couples to SPPs on the outer surface of a thin metal film deposited on an optically transparent substrate. ${ }^{16,17} \mathrm{~A}$ light source was collimated by two narrow slits and incident on the half-cylinder prism through a polarizer. Photons are coupled into SPPs on the sample at the bottom of the prism when the incoming beam is $p$-polarized, while the SPP excitation is turned off when the polarizer is turned $90^{\circ}$, producing an $s$-polarized ray. ${ }^{16}$

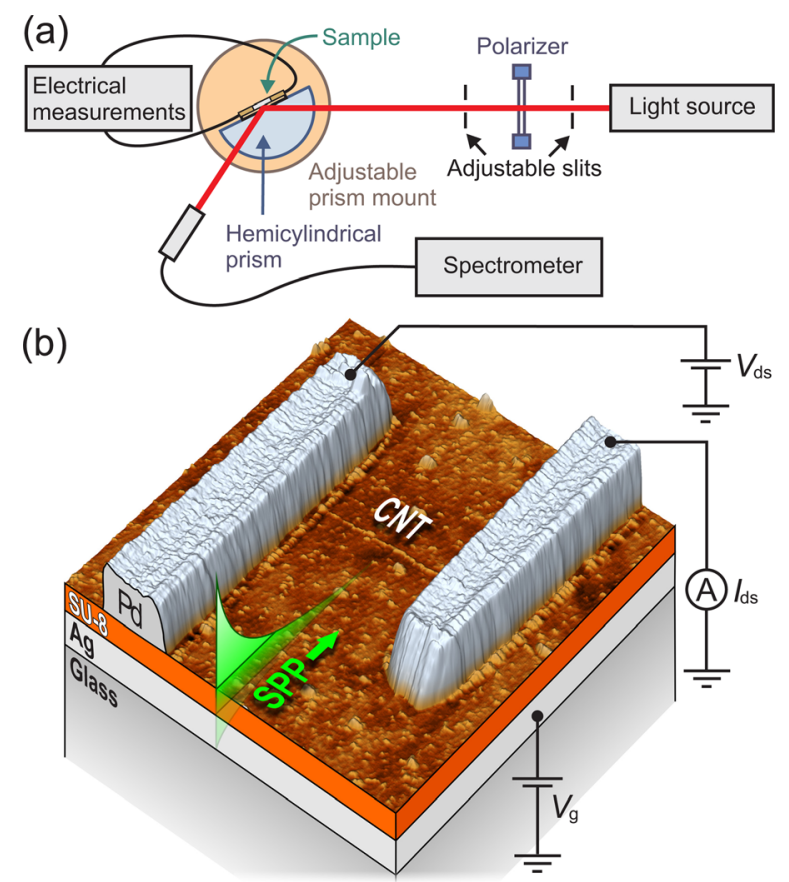

FIG. 1. (Color online) (a) Schematics of the experimental setup. (b) AFM image of the device. Edge dimensions are $2.5 \mu \mathrm{m}$ and the SPPs are excited on the interface between SU- 8 and silver in the direction of the arrow. 

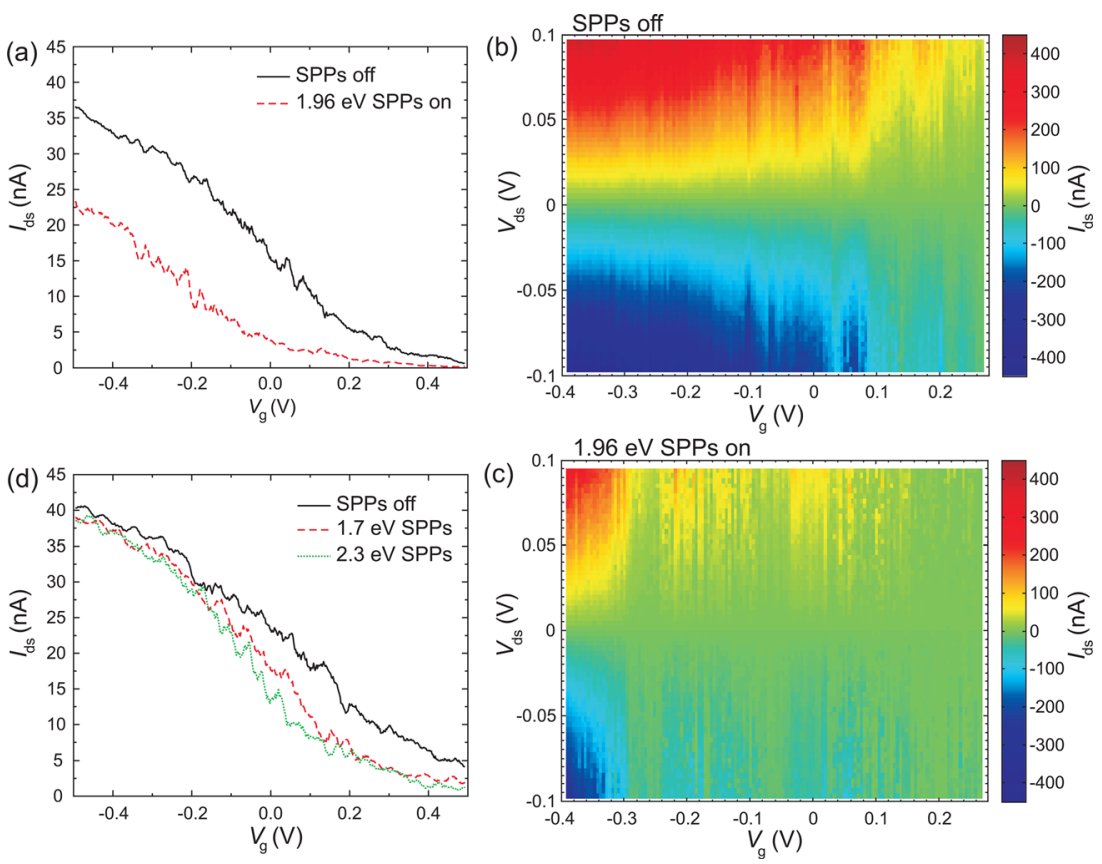

FIG. 2. (Color online) (a) Gate voltage sweeps with SPPs on and off. Four traces were averaged for each curve to decrease the noise level (negligible hysteresis). $V_{\mathrm{ds}}=10 \mathrm{mV}$. (b) Drain-source current as a function of $V_{\mathrm{g}}$ and $V_{\mathrm{ds}} .1 .96 \mathrm{eV}$ SPPs are switched off by polarization. $V_{\mathrm{ds}}$ is the fast scan axis ( $23 \mathrm{~s}$ for one line) and $V_{\mathrm{g}}$ is the slow scan axis. (c) A corresponding measurement for the same device with SPPs switched on. (d) $V_{\mathrm{g}}$ sweeps with excitation by white light and plasmon energy adjusted with the angle of incidence. $1.7 \mathrm{eV}$ excitation angle was used in the measurement with SPPs off. $V_{\mathrm{ds}}=10 \mathrm{mV}$.
This is used in the measurements as a control for possible effects due to direct light excitation; even though the sample is illuminated on the other side of the metal film compared to the CNT FET. The excited SPPs travel to the CNT FET perpendicular to the SWCNT, as shown in Fig. 1(b).

Samples were produced on a $1 \mathrm{~cm}^{2}$ glass slide. For SPPs, a $63 \mathrm{~nm}$ thick and $1 \mathrm{~mm}$ wide silver strip was evaporated on the glass followed by spin-coating with diluted SU-8 2025 (MicroChem) resist. The resulting $50 \mathrm{~nm}$ thick cured SU-8 layer acts as a dielectric between the CNT circuit and the Ag strip, which doubles as a back gate. Photolithography and electron beam lithography (EBL) were used to produce palladium electrodes and alignment markers on the SU-8 surface. ${ }^{18}$

Commercial SWCNTs (Nanocyl S.A.) were spinned from a 1,2-dichloroethane suspension on the sample surface after breaking up bundles with sonication and located in relation to the alignment markers by AFM scans. ${ }^{18}$ Finally, 84 $\mathrm{nm}$ thick Pd electrodes completing the CNT circuit were fabricated with EBL and the sample was connected to the setup.

Circularly polarized $633 \mathrm{~nm}(1.96 \mathrm{eV}) \mathrm{HeNe}$ laser (Uniphase 1125) was used as a light source producing a power density of $\sim 700 \mathrm{~mW} / \mathrm{cm}^{2}$ on the prism after polarization. The incident beam had a spot size of $0.8 \mathrm{~mm}$ in diameter, pointed at the section of the Ag film where on the opposite side, the CNT FET is located. Plasmon resonance for the beam occurred at an angle of incidence of $62^{\circ}$, and the intensity of the SPPs produced to the Ag/SU-8 interface was approximately $110 \mathrm{~mW} / \mathrm{cm}^{2}$ based on the measured reflected intensity. ${ }^{18}$ The exposed length of the $2.3 \pm 0.7 \mathrm{~nm}$ thick SWCNT was $1 \mu \mathrm{m}$ [see Fig. 1(b)].

The current, $I_{\mathrm{ds}}$, flowing through the channel of the CNT FET was simultaneously measured during excitation by applying a drain-source voltage, $V_{\mathrm{ds}}$, and a gate voltage, $V_{\mathrm{g}}$. The CNT FET was initially measured with the $s$-polarized beam (no SPPs). It was found to have a $p$-type transistor response [see Fig. 2(a)], which is typical for devices with Pd contacts in ambient conditions. ${ }^{19}$ Gate voltages greater than $\pm 0.5 \mathrm{~V}$ were not used due to the limited durability of the SU-8 layer. Notable is that the CNT FETs made with an SU-8 dielectric layer showed almost no hysteresis in the gate sweep curves (not shown). ${ }^{20}$ When the laser beam was set to allow SPP excitation ( $p$-polarized), the current response is suppressed and shifted to the left in Fig. 2(a). The contour plots in Figures 2(b) and 2(c) show clearly that a shift of about $0.4 \mathrm{~V}$ in effective gate voltage has taken place when turning the polarization of the laser $90^{\circ}$.

The temporal current response, $I_{\mathrm{ds}}$, with a constant $V_{\mathrm{ds}}$ and $V_{\mathrm{g}}$, is slow both after turning the SPPs on, inducing a drop in the conductance, and when the device is recovering after turning SPPs off. We suggest that photoinduced desorption and subsequent adsorption of molecules on the CNT FET explain the results. A likely candidate is $\mathrm{O}_{2}$, which has been shown to spontaneously $p$-dope CNTs when exposed to air. ${ }^{2,5,8}$ We cannot rule out the possibility of desorption of other molecules present in ambient conditions. However, oxygen has been shown to have the strongest influence. ${ }^{8}$ The time dependence in Fig. 3 conforms well to an exponential fit in both directions, with time constants of $131 \pm 3 \mathrm{~s}$ and $343 \pm 3 \mathrm{~s}$ for the desorption and adsorption, ${ }^{18}$ respectively, while there is no response in current to the laser excitation when it is not producing SPPs. The observed desorption rate is faster than measured for far field photodesorption with the same wavelength, ${ }^{5}$ even when taking into account our higher intensity. This is consistent with the observations on the plasmon stimulated desorption on metal, ${ }^{15}$ indicating SPP induced rate enhancement.

To study the energy dependence, the device was excited with a white light source, i.e., a tungsten-halogen lamp, and the energy of the excited SPPs was adjusted by the angle of incidence. Figure 2(d) shows the response for SPPs of two different approximate energies, ${ }^{18}$ compared to no SPPs. The overall effect is much smaller than observed with laser due to the lower intensity of the light source, i.e., $7 \mathrm{~mW} / \mathrm{cm}^{2}$. Also, it should be noted that the produced SPPs have a Gaussian spectrum centered at the resonance wavelength. The intensity coupled to SPPs was $\sim 20 \mu \mathrm{W} / \mathrm{cm}^{2}$ for both green 


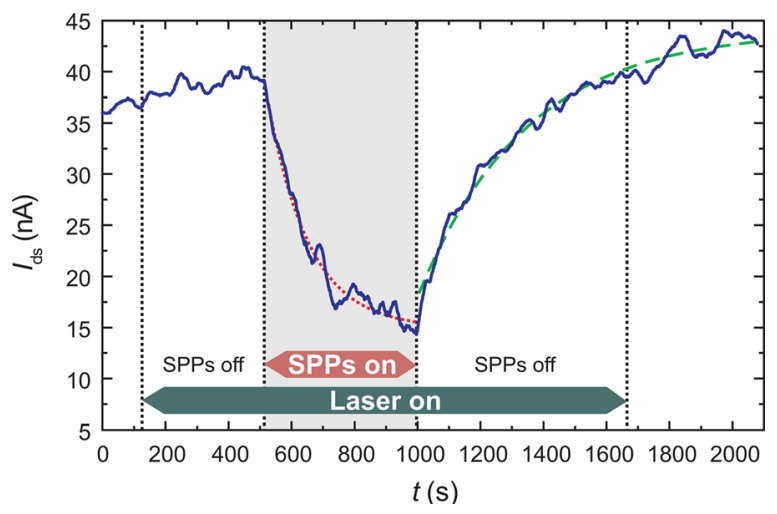

FIG. 3. (Color online) A typical measurement of the current response to laser exposure and SPP excitation controlled by polarization. Exponential fits are provided for desorption (dotted) and adsorption (dashed). $V_{\mathrm{g}}=-0.4$ $\mathrm{V}, V_{\mathrm{ds}}=10 \mathrm{mV}$.

$(2.3 \mathrm{eV})$ and near-infrared $(1.7 \mathrm{eV})$ excitation. ${ }^{18}$ We note that all these excitation energies are significantly higher than the binding energy of physisorbed oxygen, which is about $0.25 \mathrm{eV}^{21}$ However, as seen from the figure, the response was slightly larger with higher plasmon energy, which is expected behavior for a photodesorption effect. ${ }^{5}$

Direct photocurrent due to electron-hole-pair creation would give the opposite effect, a fast increase in the current response, which at no point was observed. ${ }^{3,22}$ However, the polarization of the SPPs being perpendicular to the axis of the CNT can explain this, since the photocurrent generation is maximized for light linearly polarized along the length of the CNT. ${ }^{3}$ Also, highest response is at energies corresponding to the van Hove singularities, ${ }^{23}$ so the effect should be minimal in these measurements.

If the substrate is a semiconductor such as $\mathrm{Si}$, a photovoltage can also be generated at the interface between the backgate and the dielectric, ${ }^{24}$ producing a shift in the effective gate voltage. Here, the use of a metal backgate prevents this effect, as evidenced by the lack of response for $s$-polarized laser. Direct heating of the sample by the laser is not affecting the conductivity of the CNT FET, as can be seen in Fig. 3. Neither can heating by SPPs be the reason for the observations; even a conservative estimation of temperature change in the device is less than $1 \mathrm{~K}$, changing the conductivity less than $1 \% .{ }^{18}$ Note also that such scaling of the resistance could not explain the observed shift in effective gate voltage..$^{25,26}$ In addition, it has been shown that CNTs well connected to the substrate do not show an appreciable increase in temperature for incident farfield light power densities up to $100 \mathrm{~kW} / \mathrm{cm}^{2}$.

The exact place of the molecular photodesorption, i.e., either at the CNT-metal contact region or along the CNT itself, is widely disputed. ${ }^{2}$ The switching properties of CNT FETs are generally dominated by the response of the CNT-metal contact region, resulting in a Schottky-barrier transistor instead of a bulk-switching transistor. ${ }^{4,27}$ Some studies point to strongest chemical activity on the CNT-metal contact, ${ }^{11}$ but others show doping effects of the SWCNT itself ${ }^{19}$ or are otherwise contradicting the idea of oxygen modifying the Schottky barrier via the electrode metal work function. ${ }^{28}$ Yet, CNT FETs connected with Pd electrodes are shown to have lower effect, if any at all, on the desorption on the CNT-metal interface region. ${ }^{19}$ From our data, it cannot be determined whether the desorption happens on the CNT or the CNT-metal contacts, and what type of desorption process is in question. Even though AFM imaging did not reveal obvious defects in the CNT, the processing with ultrasonication could have introduced them, providing possibly more active sites for molecular adsorption directly to SWCNT.

In summary, we demonstrated that $1.96 \mathrm{eV}$ SPPs propagating at an interface $50 \mathrm{~nm}$ below the active region of a $p$ type CNT FET significantly modulate its conductivity and induce a $0.4 \mathrm{~V}$ shift in the effective gate voltage. The conductivity diminishing at a slow rate in response to the SPP excitation, as well as the decreasing of the effect with lower SPP energy can be explained by desorption of physisorbed molecules, most likely oxygen. The effect was observed even at low intensities and in near-IR, which is contrary to direct photodesorption.

This work was supported by the Academy of Finland (Project Nos. 135193, 218182, 130039, 213362, 217045, 135000,141039 ) and conducted (see www.esf.org/euryi) as part of a EURYI scheme grant.

${ }^{1}$ D. Kauffman and A. Star, Angew. Chem. Int. Ed. 47, 6550 (2008).

${ }^{2}$ P. Bondavalli, P. Legagneux, and D. Pribat, Sens. Actuators, B 140, 304 (2009).

${ }^{3}$ M. Freitag, Y. Martin, J. A. Misewich, R. Martel, and P. Avouris, Nano Lett. 3, 1067 (2003)

${ }^{4}$ P. Avouris, J. Chen, M. Freitag, V. Perebeinos, and J. Tsang, Phys. Status Solidi B 243, 3197 (2006).

${ }^{5}$ R. Chen, N. Franklin, J. Kong, J. Cao, T. Tombler, Y. Zhang, and H. Dai, Appl. Phys. Lett. 79, 2258 (2001).

${ }^{6}$ Y. Shi, W. Fang, K. Zhang, W. Zhang, and L. Li, Small 5, 2005 (2009).

${ }^{7}$ D. McClain, N. Thomas, S. Youkey, R. Schaller, J. Jiao, and K. O'Brien, Carbon 47, 1493 (2009).

${ }^{8}$ P. Collins, K. Bradley, M. Ishigami, and A. Zettl, Science 287, 1801 (2000).

${ }^{9}$ H. Ulbricht, G. Moos, and T. Hertel, Phys. Rev. B 66, 75404 (2002).

${ }^{10}$ D. Kang, N. Park, J. Hyun, E. Bae, J. Ko, J. Kim, and W. Park, Appl. Phys. Lett. 86, 093105 (2005).

${ }^{11}$ M. Shim and G. Siddons, Appl. Phys. Lett. 83, 3564 (2003).

${ }^{12}$ W. L. Barnes, A. Dereux, and T. W. Ebbesen, Nature 424, 824 (2003).

${ }^{13}$ A. V. Zayats, I. I. Smolyaninov, and A. A. Maradudin, Phys. Rep. 408, 131 (2005).

${ }^{14}$ K. Kneipp, H. Kneipp, P. Corio, S. D. M. Brown, K. Shafer, J. Motz, L. T. Perelman, E. B. Hanlon, A. Marucci, G. Dresselhaus, and M. S. Dresselhaus, Phys. Rev. Lett. 84, 3470 (2000).

${ }^{15}$ W. Hoheisel, K. Jungmann, M. Vollmer, R. Weidenauer, and F. Träger, Phys. Rev. Lett. 60, 1649 (1988).

${ }^{16}$ L. Novotny and B. Hecht, Principles of Nano-optics (Cambridge University Press, Cambridge, 2006).

${ }^{17}$ T. K. Hakala, J. J. Toppari, A. Kuzyk, M. Pettersson, H. Tikkanen, H. Kunttu, and P. Törmä, Phys. Rev. Lett. 103, 053602 (2009).

${ }^{18}$ See supplementary material at http://dx.doi.org/10.1063/1.3614543 for sample fabrication details and plasmon resonance data.

${ }^{19}$ M. Shim, J. Back, T. Ozel, and K. Kwon, Phys. Rev. B 71, 205411 (2005).

${ }^{20}$ M. Rinkiö, A. Johansson, G. S. Paraoanu, and P. Törmä, Nano Lett. 9, 643 (2009).

${ }^{21}$ S. Jhi, S. Louie, and M. Cohen, Phys. Rev. Lett. 85, 1710 (2000).

${ }^{22}$ I. Levitsky and W. Euler, Appl. Phys. Lett. 83, 1857 (2003).

${ }^{23}$ X. Qiu, M. Freitag, V. Perebeinos, and P. Avouris, Nano Lett. 5, 749 (2005).

${ }^{24}$ M. Marcus, J. Simmons, O. Castellini, R. Hamers, and M. Eriksson, J. Appl. Phys. 100, 084306 (2006).

${ }^{25}$ E. Pop, D. Mann, Q. Wang, K. Goodson, and H. Dai, Nano Lett. 6, 96 (2006).

${ }^{26}$ M. Itkis, F. Borondics, A. Yu, and R. Haddon, Science 312, 413 (2006).

${ }^{27}$ V. Vitale, A. Curioni, and W. Andreoni, J. Am. Chem. Soc. 130, 5848 (2008).

${ }^{28}$ D. McClain, N. Thomas, S. Youkey, R. Schaller, J. Jiao, and K. O'Brien, IEEE Electron Device Lett. 31, 156 (2010). 